\title{
The Analysis of Refraction and Accommodation of Extreme Myopia with Cataract after Phacoemulsification and IOL Implantation
}

\author{
Samer Abdo Al-Wesabi ${ }^{1}$, Ruibo Yang ${ }^{1}$, Gaiyun Li ${ }^{2}$, Abdulsalam M. Nasser ${ }^{3}$, \\ Shaozhen Zhao ${ }^{1}$ \\ ${ }^{I}$ Department Of cornea and refractive surgery, Tianjin Medical University Eye hospital, Tianjin Medical \\ University, Tianjin, China. \\ ${ }^{2}$ Department Of Ophthalmology, Tongji Hospital, Tongji Medical College, Huazhong University Of Science And \\ Technology, Wuhan, China. \\ 3 Department Of Health Management, Tongji Medical College, Huazhong University Of Science And \\ Technology, Wuhan, China.
}

\begin{abstract}
:
Background: To assess the refractive status of cataract patients with extreme myopia and to measure their accommodative capability after monofocal IOL implantation.

Design: Prospective non-randomized study conducted at Tianjin Medical University Eye Centre.

Participants: 18 patients with 34 extreme myopic eyes ( $\geq-10$ Diopter, $\geq 27 \mathrm{~mm}$ axial length), and 14 patients with 27 non-myopic eyes that had uneventful phacoemulsification and monofocal IOL implantation.

Methods: The accommodation amplitude of the two groups was compared pre- and post-operatively using subjective minus-lenses-to-blur method. In the extreme myopic group, the postoperative target refraction was selected to be myopic, and they were evaluated for predicted postoperative spherical power consistency. Extreme myopic eyes anterior chamber depth was measured by pentacam, and axial length was measured by the immersion vector A/B-scan biometry. All measurements were done preoperatively and at 1, 3, 6 months postoperatively.
\end{abstract}

Main Outcome Measures: Predicted spherical power, amplitude of accommodation, anterior chamber depth, axial length.

Results: There was a significant difference between pre and postoperative results of Mean predicted spherical power $(P=0.001)$. The differences between pre- and postoperative anterior chamber depth measurements were statistically significant $(P=0.001)$. No significant differences were detected between pre- and postoperative measurements of axial length $(P>0.05)$. There was no significant difference in amplitude of accommodation between the two groups pre or postoperatively ( $P>0.05)$.

Conclusions: The study yielded a hyperopic shift of the predicted postoperative spherical power of extreme myopic eyes. No significant difference was observed between extreme myopic and non-myopic eyes in accommodation amplitude pre- or post-operatively.

Key words: extreme myopia, refractive status, apparent accommodation, cataract, phacoemulsification.

Running head: Refraction and Accommodation of Extreme Myopia 


\section{Introduction}

Cataract surgery solves both visual and refractive problems in patients with cataract and high myopia. Phacoemulsification is a safe and reliable surgery for restoration of visual acuity in patients with cataract, and is superior to extracapsular cataract extraction (ECCE). ${ }^{1}$

However, the refractive outcome is also less predictable in highly myopic eyes due to inaccuracies in axial length (AL) measurement in the presence of posterior staphyloma and poor fixation from macular disease. ${ }^{2}$ It has been accepted that monofocal IOL implantation corrects visual acuity for either distance or near vision. However, patients with monofocal IOL implantation occasionally due to apparent accommodation, have good uncorrected visual acuity (UCVA) for distance and near vision., ${ }^{3,4}$

To further assess the outcomes of extreme myopia with cataract patients, a prospective randomized controlled study was investigated on eyes with a refraction $\geq-10 \mathrm{D}$ and $\mathrm{AL} \geq 27.0 \mathrm{~mm}$ that had phacoemulsification and implanted with monofocal IOL. Particular attention was directed toward the difference between the "actual" and "predicted" postoperative spherical refractive power with its influence factors, and the accommodation amplitude in comparison with non- myopic eyes.

\section{Patients and Methods}

\section{Patients}

This study comprised 61 eyes of 32 consecutive patients who underwent uneventful phacoemulsification and monofocal IOL implantation performed by the same surgeon at department of cornea and refractive surgery, Tianjin Medical University Eye hospital, Tianjin Medical University, Tianjin, China, between March and September 2011. The current study has performed according to the tenets of the Declaration of Helsinki. In addition, the ethics committee approved the protocol of the study.

Patients' characteristics are shown in Table 1.

All patients had significant lens opacity; none of them had a prior history of ocular trauma, eye surgery, intraocular inflammation, glaucoma, or diabetes. Non-myopic eyes had no remarkable retinal abnormality. All extreme myopic eyes had a posterior staphyloma, and the retinal abnormality included degenerative myopic maculopathy in (10 eyes, 30\%), epi-retinal macular membrane (4 eyes, 12\%), and macular hard drusen (3 eye, $9 \%)$.

\section{Methods}

Visual acuity was recorded with the help of internally illuminated standard Snellen's Chart and recorded as decimal values. For the IOL power calculation, SRKII calculation formula was used for the assessments in non-myopic eyes, while SRK/T formula was used in extreme myopic eyes. In the extreme myopic group, the postoperative target refraction was selected to be myopic, with the patients' agreement; for best post-operation near vision without the need of glasses. In non-myopic group, the postoperative target refraction was between plano and $-0.50 \mathrm{D}$.

Extreme myopic eyes corneal curvature and anterior chamber depth (ACD) were measured with the pentacam comprehensive eye scanner (Pentacam CES; Oculus GmbH, Wetzlar, Germany). The axial length (AL) measurement was performed by the immersion vector A/B-scan biometry (Quantel medical, France).

Apparent accommodation was measured with the subjective minus-lenses-to-blur method. After the correction of near vision over the best corrected distance vision (BCDVA), the reading target (a Jaeger chart where the patient was asked to focus on the line just above the lowest discernable line) was placed $0.33 \mathrm{~m}$ away from the eye. $-0.25 \mathrm{D}$ spherical lenses were sequentially placed in front of the eye until the patient reported target line could no longer be held in sharp focus. The accommodative power was the absolute value of the sum of the diopters of all minus lenses which added until the target blurred, then 3 Diopters added, which corresponds to 
the accommodative effort needed to clearly view the target at a distance of $33 \mathrm{~cm}$, then the diopters of the correction of near vision was subtracted.

Measurements of $\mathrm{ACD}, \mathrm{AL}$ and corneal curvature were repeated for extreme myopic eyes and measurement of apparent accommodation was repeated for both groups at 1, 3 and 6 months postoperatively.

\section{Surgical Procedures}

All surgeries were performed by the same consultant surgeon and were conducted under topical anaesthesia. All operations were performed through a 3-mm superior clear corneal incision. A foldable posterior chamber intraocular lens (AcrySof ${ }^{\circledR}$ SN60AT single-piece) was implanted in the capsular bag after the removing of the lens matter using phacotechnology (Infiniti, Alcon Laboratories Inc.).

\section{Statistical Analysis}

All statistical calculations were done using SPSS ver. 11.5. Results were compared using one way ANOVA with repeated measures. Normality for continued variables in a group was determined by Shapiro-Wilks test. When a normal distribution was not expected; Friedman test was done, and post hoc analysis was used to determine whether there were pairwise differences. Independent T-Test was used to compare between the two groups. Results are expressed as means \pm standard deviation and a P value less than 0.05 was considered statistically significant.

\section{Results}

All eyes were received positive power IOL. Patients were followed for 6 months after the surgery; no patient missed a scheduled examination. Results of pre and postoperative uncorrected and corrected visual acuity for extreme myopic group and non-myopic group are demonstrated in Table. 2 and Table. 3 respectively. Outcomes of $\mathrm{ACD}, \mathrm{AL}$ and corneal curvature of extreme myopia are as following:

Mean ACD measurements were $3.10 \pm 0.27 \mathrm{~mm}$ preoperatively and $3.42 \pm 0.25 \mathrm{~mm}, 3.42 \pm 0.24 \mathrm{~mm}$ and $3.43 \pm 0.21 \mathrm{~mm}$ at 1,3 and 6 months postoperatively, respectively. The differences between pre- and postoperative ACD measurements were statistically significant $P=0.000$. Mean keratometric horizontal meridian measurements were $43.25 \pm 0.97 \mathrm{D}$ preoperatively, and $43.25 \pm 1.07 \mathrm{D}, 43.17 \pm 1.05 \mathrm{D}$ and $43.25 \pm 1.18 \mathrm{D}$ at 1,3 and 6 months postoperatively, respectively. The differences between pre- and postoperative measurements were not statistically significant $P=0.66$. Mean keratometric vertical meridian measurements were $44.10 \pm 1.65 \mathrm{D}$ preoperatively, and $44.25 \pm 1.40 \mathrm{D}, 44.22 \pm 1.35 \mathrm{D}$ and $44.18 \pm 1.29 \mathrm{D}$ at 1,3 and 6 months postoperatively, respectively. The differences between pre- and postoperative measurements were not statistically significant $P=$ 0.36. Mean AL measurements were $30.05 \pm 1.96 \mathrm{~mm}$ preoperatively, and $30.05 \pm 1.89 \mathrm{~mm}, 30.06 \pm 1.90 \mathrm{~mm}$ and $30.06 \pm 1.86 \mathrm{~mm}$ at 1,3 and 6 months postoperatively, respectively. The differences between pre- and postoperative measurements were not statistically significant $P=0.85$.

Mean predicted spherical power of extreme myopic eyes was $-3.66 \pm 0.50 \mathrm{D}$ preoperatively, whilst mean postoperative achieved spherical powers were $-3.25 \pm 0.63 \mathrm{D},-3.23 \pm 0.68 \mathrm{D}$ and $-3.20 \pm 0.65 \mathrm{D}$ at 1,3 and 6 months respectively. There were a significant difference between pre and postoperative results $P=0.000$. No statistically significant difference among the group postoperatively $P>0.05$.

There was no statistically significant difference in amplitude of accommodation between extreme myopic and non-myopic pre and postoperatively (Fig.1). Preoperative accommodation and postoperative apparent accommodation outcomes are demonstrated in Table.4.

\section{Discussion}

This study attempted to assess the refractive status of post phacoemulsification cataract patients with extreme myopia, and to detect their pseudo-accommodative capability after monofocal IOL implantation. Lee at 
el. ${ }^{5}$ has defined extreme myopia (super high myopia) as Myopia $\geq-10 \mathrm{D}$. Percival SP. ${ }^{6}$ suggested that a definition of high myopia based on axial length is more appropriate than that based on refraction; however, nuclear cataract usually causes a myopic shift in refraction. ${ }^{7}$ In this study, an axial length of $\geq 27 \mathrm{~mm}$ and refraction of $\geq-10 \mathrm{D}$ were chosen for the inclusion criteria because we believe that this is a better estimation for extreme myopia.

The optimum post-cataract refraction should be based on the patient's visual needs, occupation, and lifestyle. In this study, in consideration of a convenient life style and patient's visual needs, extreme myopic eyes were managed to be myopic postoperatively $(-3.00$ to -5.00$)$ for good postoperative near vision. Eighty tow percent of extreme myopic eyes achieved a BCVA of 0.5 or better which is relatively higher than that was reported by Zuberbuhler et al. ${ }^{8}$ and comparable with that reported by Tsai et al. ${ }^{9}$ Pre-existing high myopic maculopathy is an independent risk factor for poor postoperative vision. ${ }^{9}$

Postoperative results of this study showed a significant deviation (hyperopic shift) from pre-operation predicted spherical power. Many studies reported that the SRK/T formula, which we used for IOL power calculation, is appropriate for biometry in high myopia of $\geq 27 \mathrm{~mm}$ axial length. ${ }^{2,8,10}$ Zaldivar et al. ${ }^{11}$ reported that $92 \%$ of eyes were within $\pm 1.0 \mathrm{D}$ when using SRK/T, and concluded that in eyes with axial lengths $\geq 27.0$ $\mathrm{mm}$, current third- and fourth-generation lens calculation formulas have a tendency to over minus patients between -1.0 and $-4.0 \mathrm{D}$, leaving patients with postoperative hyperopia. Similarly, Tsang et al. ${ }^{2}$ found that SRK/T formula showed a slight tendency toward resultant hyperopia, resulting in a postoperative hyperopic refractive error.

Generally, there is a difficulty in measuring AL for extreme myopic eyes; this partly may be due to the pathological changes of posterior pole anatomy. The fovea is approximately $4.5 \mathrm{~mm}$ ( 3 disc diameter or $15^{\circ}$ ) from the center of the optic nerve. Holladay and Prager ${ }^{12}$ have performed high resolution B-scans with the innovative imaging system using horizontal sections through the optic nerve and measured distance from the corneal vertex to a point $4.5 \mathrm{~mm}$ temporal to the center of the optic nerve, they found that in eyes with axial lengths $\geq 30.0 \mathrm{~mm}$, a posterior pole staphyloma temporal to the fovea was common, and the corneal vertex-fovea distance was approximately $0.5-1.5 \mathrm{~mm}$ shorter than the distance from the corneal vertex to the bottom of the staphyloma, which is where the A-scan usually finds the perpendicular axis and records the axial length. This may lead to inaccuracy of AL measurement, and hence, may affect precise IOL power calculation.

In the current study, no significant changes of AL measurement were detected pre or postoperatively. However, the immersion B-scan/vector A-scan has the advantage of the operator being able to direct the axial length measurement to the region of the fovea, giving the refractive, rather than the anatomic axial length. For eyes with high axial myopia and peripapillary posterior staphyloma, this is the preferred biometry technique by ultrasound that has a better consistency than the immersion A-scan technique, or the applanation method which indents the cornea resulting in corneal compression. Another factor could influence the postoperative refraction of high myopia, is ACD. The result of this study showed a significant increase of ACD postoperatively. The thickness of the IOL is highly less than the crystalline lens thickness. After the IOL implantation in the capsular bag, the anterior chamber become deeper, the distance from the cornea to where the seen object should be reflected on the IOL surface is longer than that on the crystalline lens surface. This could push the reflection image of an object slightly behind the retina, and cause a slight hyperopic shift of the actual precise refraction.

The measurements of ACD did not change significantly at the 1-3- and 6-month examinations postoperatively, which was in accordance with that has been reported by Doganay et al. ${ }^{13}$ That means the values of anterior chamber depth remained stable after one month of phacoemulsification. There were no significant changes of corneal curvature measurements by pentacam preoperatively and over the 6 months postoperatively.

Nakazawa $\mathrm{M}$ and Ohtsuki $\mathrm{K}^{5}$ measured 39 eyes of 31 patients (age was $42-90$ years) who underwent 
cataract extraction and posterior chamber lens implantation, and the mean accommodative power was $2.03 \pm 1.03 \mathrm{D}$. Similarly, Yang et al. ${ }^{14}$ found that the mean accommodative power of pseudophakic was $2.15 \pm 0.76 \mathrm{D}$. Results of this study were comparable with those above mentioned studies, whereas, somehow higher than that reported by Nemeth at el. ${ }^{15}$ who used minus-lenses-to-blur reporting a mean of $0.83 \pm 0.63 \mathrm{D}$.

Amplitude of accommodation (AoA) measurements reflect the maximum capacity a subject has to stimulate his accommodation response. The minus-lenses-to-blur is one of the most widely used subjective methods for assessing the AoA. It requires that the subject correctly identify the power at which the target fixed on becomes and stays blurred. So it depends on patient cooperation and understanding. Subjective methods were found to be more reliable for measuring apparent accommodation according to Langenbucher et al. ${ }^{16}$ Many previous researches confirmed that the amplitude of apparent accommodation is correlated with different factors including; depth of focus ${ }^{17,18}$, pupil diameter ${ }^{4,19}$, degree of corneal astigmatism ${ }^{20}$, corneal multifocality and aberrations ${ }^{21,22}$, and IOL axial forward movement. ${ }^{23,24}$ Depth of focus is an intrinsic characteristic of all optical systems that was considered to be one of the most significant factors of apparent accommodation. Nakazawa and $\mathrm{Ohtsuki}^{4}$ demonstrated that the amount of apparent accommodation is highly correlated to the calculated depth of focus in eyes implanted with monofocal IOLs. Depth of focus correlates to normal levels of visual acuity, the higher the visual acuity, the lower the depth of focus. ${ }^{25}$

Axial movement of the IOL is a factor that has also been studied as a contributing factor to pseudoaccommodation of monofocal IOLs. It depends on the range of movement of the IOL and the position of the IOL. ${ }^{26}$ One study calculated that the accommodation obtained per $1.0 \mathrm{~mm}$ of forward IOL movement could reach $0.8 \mathrm{D}$ in long axial eyes. ${ }^{27}$ Another study used the subjective near point as the stimulus found a forward IOL movement of $0.04 \mathrm{~mm}$ in pseudophakic eyes during accommodation. ${ }^{28}$

It has been assumed that myopic eyes accommodation ability is less than emmetropic eyes particularly around the presbyopic duration of life; most of the published studies in accommodation amplitude exclude high myopia off their subjects. However, according to our result, there was no significant difference between extreme myopic and non-myopic eyes in accommodation amplitude pre- or post-operatively.

Park et al. ${ }^{29}$ have shown that, although there is a decrease in contractility of ciliary body with age in phakic patients, contractility increases after cataract surgery. Strenk et al. ${ }^{30}$ have shown with magnetic resonance imaging that the ciliary muscle remains active throughout life, although there are age-related changes that may interfere with presbyopia. Stachs et al. ${ }^{31}$ also demonstrated with the use of ultrasound that the ciliary body is active in the presbyopic age. Another study showed that during accommodative stimuli in presbyopic pseudophakic eyes, there are variations in anterior segment structures similar to those in phakic prepresbyopic eyes. In particular, there is an anteroposterior rotation with simultaneous centripetal shift of ciliary bodies and processes, causing a reduction in sulcus and capsular bag diameter. These movements influence the IOL position, which undergoes modification during accommodative stimuli. ${ }^{32}$

It is known that with eye axial elongation, many structures of the eye happen to have accompanied change including ciliary body and posterior iris. However, a study of ciliary muscle morphologic changes with accommodation in vivo deduced that the ciliary muscle was not found to be attenuated in myopic subjects as would be predicted from stretching alone, in fact, no significant relationship between ciliary muscle thickness and refractive error was identified. ${ }^{33}$ It seems therefore, that axial elongation is accompanied by some radial growth - thickening of the ciliary muscle during myopigenesis.

\section{Conclusion}

This study investigated refractive status and accommodation amplitude of extreme myopic eyes that combined with cataract after phacoemulsification and monofocal non-accommodative IOL implantation. The 
result of current study showed a biometry error and hyperopic shift of predicted postoperative spherical power, which is consistent with what have been reported by previous studies. No significant difference was observed between extreme myopic and non-myopic eyes in accommodation amplitude pre- or post-operatively. There was a significant increase of ACD postoperatively. No significant change was observed between pre and postoperative corneal curvature. To our knowledge this is the first study evaluating accommodation amplitude in extreme myopia. Further studies are suggested with larger sample size and longer follow up duration.

\section{References}

[1]. Cheng JW, Wei RL, Li Y. Effect of phacoemulsification versus extracapsular extraction on visual acuity: a meta-analysis. Zhonghua Yan Ke Za Zhi 2004; 40: 474-7.

[2]. Tsang CSL, Chong GSL, Yiu EPF, Ho CK. Intraocular lens power calculation formulas in Chinese eyes with high axial myopia. J Cataract Refract Surg 2003; 29:1358-1364.

[3]. Nanavaty MA, Vasavada AR, Patel AS, Raj SM, Desai TH. Analysis of patients with good uncorrected distance and near vision after monofocal intraocular lens implantation. J Cataract Refract Surg 2006 Jul; 32 (7):1091-7.

[4]. Nakazawa M, Ohtsuki K. Apparent accommodation in pseudophakic eyes after implantation of posterior chamber intraocular lenses. Am J Ophthalmol 1983; 96:435-38.

[5]. Lee JS, Oum BS, Lee BJ, Lee SH. Photorefractive keratectomy for astigmatism greater than -2.00 diopters in eyes with low, high, or extreme myopia. J Cataract Refract Surg 1994, 24:1456-63.

[6]. Percival SP: Redefinition of high myopia. the relationship of axial length measurement to myopic pathology and its relevance to cataract surgery. Dev Ophthalmol 1987; 14: 42-46.

[7]. Panchapakesan J, Rochtchina E, Mitchell P. Myopic refractive shift caused by incident cataract: the Blue Mountains Eye Study. Ophthalmic Epidemiol 2003; 10: 241-47.

[8]. Zuberbuhler B, Seyedian M, Tuft S. Phacoemulsification in eyes with extreme axial myopia. J Cataract Refract Surg 2009; 35:335-40.

[9]. Tsai CY, Chang TJ, Kuo LL, Chou P, Woung LC. Visual outcomes and associated risk factors of cataract surgeries in highly myopic Taiwanese. Ophthalmologica 2007; 221:18-23.

[10]. Wang JK, Hu CY, Chang SW. Intraocular lens power calculation using the IOLMaster and various formulas in eyes with long axial length. J Cataract Refract Surg 2008; 34:262-67.

[11]. Zaldivar R, Shultz MC, Davidorf JM, Holladay JT. Intraocular lens power calculations in patients with extreme myopia. J Cataract Refract Surg 2000; 26:668-74.

[12]. Holladay JT, Prager TC. Accurate ultrasonic biometry in pseudophakia. Am J Ophthalmol. 1989; 107:189-90.

[13]. Doganay S, Bozgul Firat P, Emre S, Yologlu S. Evaluation of anterior segment parameter changes using the Pentacam after uneventful phacoemulsification. Acta Ophthalmol 2010 Aug; 88(5):601-6.

[14]. Yang Y, Yao K, Jiang J. Accommodation in pseudophakic eyes with monofocal posterior chamber intraocular lens. Zhonghua Yan Ke Za Zhi 1996; 32: 98-100.

[15]. Nemeth G, Tsorbatzoglou A, Vamosi P, Sohajda Z, Berta A. A comparison of accommodation amplitudes in pseudophakic eyes measured with three different methods. Eye (Lond). 2008 Jan; 22(1):65-9.

[16]. Langenbucher A, Huber S, Nguyen NX, Seitz B, Gusek-Schneider GC, Kuchle M. Measurement of accommodation after implantation of an accommodating posterior chamber intraocular lens. J Cataract Refract Surg 2003; 29: 677-85.

[17]. Elder MJ, Murphy C, Sanderson GF. Apparent accommodation and depth of field in pseudophakia. J Cataract Refract Surg 1996; 22: 615-19.

[18]. Niessen AGJE, de Jong LB, van der Heijde GL. Pseudoaccommodation in pseudophakia. Eur J Implant Refract Surg 1992; 4: 91-94.

[19]. Ravalico G, Baccara F. Apparent accommodation in pseudophakic eyes. Acta Ophthalmol 1990; 68: 604-06.

[20]. Trindade F, Oliveira A, Frasson M. Benefit of against-the-rule astigmatism to uncorrected near acuity. J Cataract Refract Surg. 1997; 23(1):82-5.

[21]. Fukuyama M, Oshika T, Amano S, Yoshitomi F. Relationship between apparent accommodation and corneal multifocality in pseudophakic eyes. Ophthalmology 1999; 106:1178-81.

[22]. Oshika T, Mimura T, Tanaka $\mathrm{S}$ et al. Apparent accommodation and corneal wavefront aberration in pseudophakic eyes. Invest Ophthalmol Vis Sci 2002 Sep; 43:2882-86.

[23]. Lesiewska-Junk H, Kaluzny J. Intraocular lens movement and accommodation in eyes of young patients. J Cataract Refract Surg 2000; 26: 562-65.

[24]. Findl O, Kiss B, Petternel V et al. Intraocular lens movement caused by ciliary muscle contraction. J Cataract Refract Surg. 2003 Apr; 29(4):669-76. 
[25]. Sergienko NM, Kondratenko YN, Tutchenko NN. Depth of focus in pseudophakic eyes. Graefes Arch Clin Exp Ophthalmol. 2008 Nov; 246(11):1623-7.

[26]. Atchison DA. Pseudoaccommodation with forward movement of IOLs. J Cataract Refract Surg. 2005 Jan; 31(1):11.

[27]. Nawa $\mathrm{Y}$, Ueda T, Nakatsuka $\mathrm{M}$ et al. Accommodation obtained per $1.0 \mathrm{~mm}$ forward movement of a posterior chamber intraocular lens. J Cataract Refract Surg. 2003 Nov; 29(11):2069-72.

[28]. Noda H, Nishida S, Ogino N et al. Objective evaluation of apparent accommodation of pseudophakic eyes. Nihon Ganka Gakkai Zasshi. 1994 Feb; 98(2):187-91.

[29]. Park KA, Yun JH, Kee C. The effect of cataract extraction on the contractility of ciliary muscle. Am J Ophthalmol. 2008 Jul; 146(1):8-14.

[30]. Strenk SA, Strenk LM, Guo S. Magnetic resonance imaging of aging, accommodating, phakic, and pseudophakic ciliary muscle diameters. J Cataract Refract Surg. 2006 Nov; 32(11):1792-8.

[31]. Stachs O, Martin H, Kirchhoff A, Stave J, Terwee T, Guthoff R. Monitoring accommodative ciliary muscle function using three-dimensional ultrasound. Graefes Arch Clin Exp Ophthalmol. 2002 Nov; 240(11):906-12.

[32]. Marchini G, Pedrotti E, Modesti M, Visentin S, Tosi R. Anterior segment changes during accommodation in eyes with a monofocal intraocular lens: high-frequency ultrasound study. J Cataract Refract Surg. 2008 Jun; 34(6):949-56.

[33]. Sheppard AL, Davies LN. In vivo analysis of ciliary muscle morphologic changes with accommodation and axial ametropia. Invest Ophthalmol Vis Sci. 2010 Dec; 51(12):6882-9.

\begin{tabular}{|c|c|c|}
\hline \multicolumn{3}{|l|}{ Table.1 Patients' characteristics } \\
\hline & Extreme myopia & Non-myopia \\
\hline Patients & 18 & 14 \\
\hline Eyes & 34 & 27 \\
\hline Gender (male/female) & $3 / 15$ & $8 / 6$ \\
\hline \multicolumn{3}{|l|}{ Age (year) } \\
\hline Mean $\pm \mathrm{sd}^{\dagger}$ & $\begin{array}{ll} & 54.94 \pm 8 . \\
91 & \end{array}$ & $\begin{array}{l}57.92 \pm 7 . \\
68\end{array}$ \\
\hline Range & $42 \sim 69$ & $45 \sim 70$ \\
\hline \multicolumn{3}{|l|}{ Axial length (millimeter) } \\
\hline Mean $\pm \mathrm{sd}^{\dagger}$ & $96 \quad 30.05 \pm 1$. & $\begin{array}{l}23.29 \pm 0 . \\
82\end{array}$ \\
\hline Range & $\begin{array}{l}27.07 \sim \\
35.3\end{array}$ & $\begin{array}{l}22.07 \sim \\
24.8\end{array}$ \\
\hline \multicolumn{3}{|l|}{ Preoperative spherical power (Diopter) } \\
\hline$\pm \mathrm{sd}^{\dagger} \quad$ Mean & 73 & $6^{0.27 \pm 1}$ \\
\hline Range & $\begin{array}{l}-10.0 \sim \\
-27.0\end{array}$ & $\begin{array}{r}-3.0 \sim \\
2.75\end{array}$ \\
\hline${ }^{\dagger}$ sd: standard deviation. & & \\
\hline
\end{tabular}

Table.2 shows uncorrected visual acuity (UCVA) outcomes

\begin{tabular}{|l|l|l|l|l|}
\hline & Preoperative UCVA & \multicolumn{3}{|c|}{ Postoperative UCVA } \\
\hline & & 1 month & 3 months & 6 months \\
\hline Extreme myopia & $0.07 \pm 0.05$ & $0.34 \pm 0.16$ & $0.33 \pm 0.17$ & $0.33 \pm 0.16$ \\
\hline Non-myopia & $0.23 \pm 0.12$ & $0.80 \pm 0.15$ & $0.82 \pm 0.16$ & $0.82 \pm 0.16$ \\
\hline
\end{tabular}




\begin{tabular}{|l|l|l|l|l|}
\hline Table.3 shows best corrected visual acuity (BCVA) outcomes \\
\hline & Preoperative BCVA & \multicolumn{4}{|c|}{ Postoperative BCVA } \\
\hline & & 1 month & 3 months & 6 months \\
\hline Extreme myopia & $0.42 \pm 0.20$ & $0.69 \pm 0.18$ & $0.70 \pm 0.17$ & $0.70 \pm 0.17$ \\
\hline Non-myopia & $0.53 \pm 0.16$ & $0.88 \pm 0.12$ & $0.88 \pm 0.12$ & $0.88 \pm 0.12$ \\
\hline
\end{tabular}

\begin{tabular}{|c|c|c|c|}
\hline & extreme myopic & non-myopic & P-value \\
\hline preoperative & $3.19 \pm 1.34$ & $2.85 \pm 0.82$ & 0.23 \\
\hline post-op 1 month & $1.27 \pm 0.72$ & $1.17 \pm 0.53$ & 0.56 \\
\hline post-op 3 months & $1.47 \pm 0.61$ & $1.31 \pm 0.56$ & 0.32 \\
\hline post-op 6 months & $1.64 \pm 0.51$ & $1.44 \pm 0.58$ & 0.18 \\
\hline
\end{tabular}

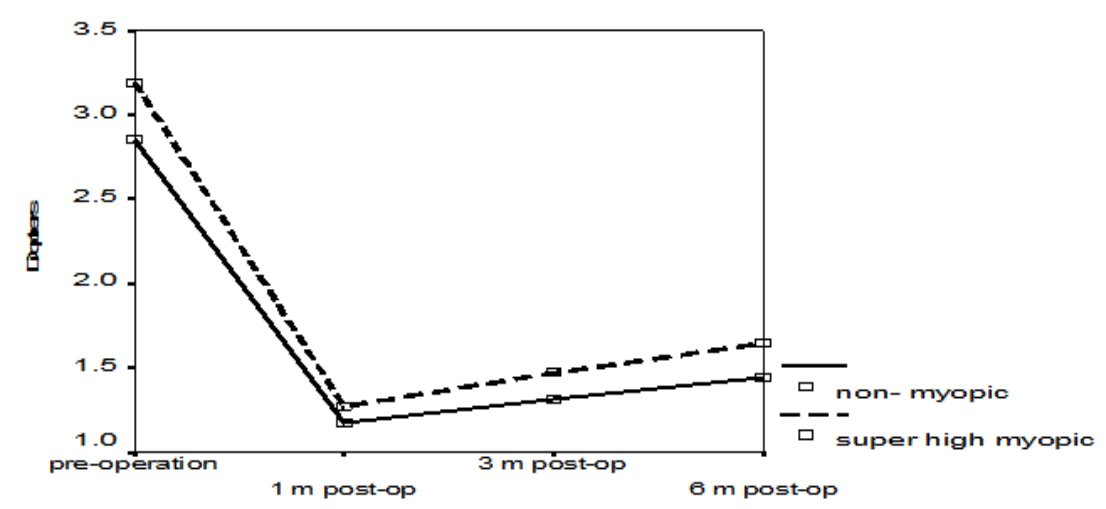

Figure 1. Shows pre and post operation mean of accommodation amplitude of the two groups. 